\title{
Askin's tumor: 11 cases and a review of the literature
}

\author{
KE ZHANG ${ }^{1}$, RUIJUAN LU ${ }^{2}$, PAN ZHANG ${ }^{3}$, SHUJING SHEN $^{4}$ and XINGYA LI ${ }^{1}$ \\ ${ }^{1}$ Department of Internal Medicine-Oncology, First Affiliated Hospital of Zhengzhou University, Henan 450000; \\ ${ }^{2}$ Department of Mental Disease, Puyang Country Mental Hospital; ${ }^{3}$ Department of Nuclear Medicine, \\ People's Hospital of Puyang, Henan 457000; ${ }^{4}$ Department of Radiotherapy, \\ First Affiliated Hospital of Zhengzhou University, Henan 450000, P.R. China
}

Received October 2, 2014; Accepted June 11, 2015

DOI: $10.3892 / \mathrm{ol} .2015 .3902$

\begin{abstract}
Askin's tumor is a peripheral primitive neruoectodermal tumor within the thoracopulmonary region, which primarily occurs in children and young adults. In addition, Askin's tumor is commonly misdiagnosed, as it is rare and easily mistaken for other small round-cell tumors. The present study aimed to investigate the clinical characteristics, prognostic factors and treatment outcomes of patients diagnosed with Askin's tumor. Computed tomography (CT) scans, histopathology and immunohistochemical analysis were used for diagnosis. Patients were treated with combined (surgery-chemotherapy-radiotherapy) or mono-therapy (chemotherapy or radiotherapy) methods. A total of 11 consecutive patients with Askin's tumor (aged 8-22 years) were treated at the First Affiliated Hospital of Zhengzhou University between April 2010 and June 2013; nine patients underwent combined therapy and two patients were treated using mono-therapy. Chest lumps, swelling and pain were the most common presenting symptoms. Patients were followed up for $\leq 24$ months post surgery and the results revealed that the median survival time of the combined and mono-therapy treatment groups were 15 and 7 months, respectively. Primary tumor size, metastasis, lactate dehydrogenase indicators and tumor stages were found to be important prognostic factors affecting patient outcome. In conclusion, the results of the present study demonstrated that the combination of surgery, chemotherapy and radiotherapy resulted in the optimal outcome for Askin's tumor patients.
\end{abstract}

\section{Introduction}

Peripheral primitive neuroectodermal tumor (PNET) is a type of soft tissue sarcoma, described as arising intracranially $(1,2)$. PNETs of the chest wall were originally reported by Askin et al

Correspondence to: $\operatorname{Dr}$ Xingya Li, Department of Internal Medicine-Oncology, First Affiliated Hospital of Zhengzhou University, 1 Jianshe Donglu, Zhengzhou, Henan 450000, P.R. China E-mail: tougao_15@163.com

Key words: Askin's tumor, combined modality, prognostic factors in 1979 (3); since then, a PNET that occurs within the thoracopulmonary region is named an Askin's tumor. Askin's tumor has been reported to primarily occur in children and young adults (3). In addition, it is a highly misdiagnosed and rare disease with a lack of clinical and pathological morphology in nature, which is easily confused with other small round-cell tumors (3). Given the rarity of this disease, no regimen has been validated for its treatment. The present study aimed to evaluate the clinical characteristics, prognostic factors and treatment outcomes of 11 cases of Askin's tumor, using clinical data as well as histopathological and immunohistochemical analysis.

\section{Materials and methods}

Patient's description. In the present study, a retrospective analysis was performed of 11 cases of pathological Askin's tumor. Patients were treated at the First Affiliated Hospital of Zhengzhou University between April 2010 and June 2013. These patients included three males and eight females, with a mean age of 14.5 years old and a range of 8 to 22 years old. All the patients were pathologically confirmed and the stages were recorded as follows: Stage I-III, six cases; stage IV, two cases; right pleural huge Askin's tumor (stage III), one case, and left side of the chest wall Askin's tumor stage II, two cases. This classification adopted the staging of soft tissue sarcoma established by American Joint Committee on Cancer in 2010 (4). The present study was approved by the ethnics committee of the First Affiliated Hospital of Zhengzhou University, Henan, China.

Diagnostics. All patients were admitted to the First Affiliated Hospital of Zhengzhou University with different degrees of symptoms, including fever, cough with progressive increase of chest pain, chest tightness and shortness of breath, certain patients presented with chest pain and chest wall tumor progressive enlargement, among other symptoms. Following admission, patients underwent chest computed tomography (CT) examination. Postoperative specimens obtained by biopsy embedded in paraffin were sliced into sections and underwent hematoxylin-eosin (HE) staining, periodic acid-Schiff staining and immunohistochemistry with antibodies for CD99, vimentin, neuron-specific enolase (NSE), S-100, creatine kinase (CK), leucocyte common antigen (LCA), myoglobin were performed. Lactate dehydrogenase (LDH) levels were also detected. 
A

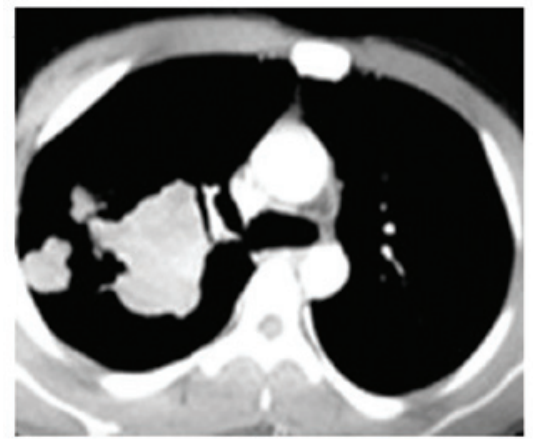

D

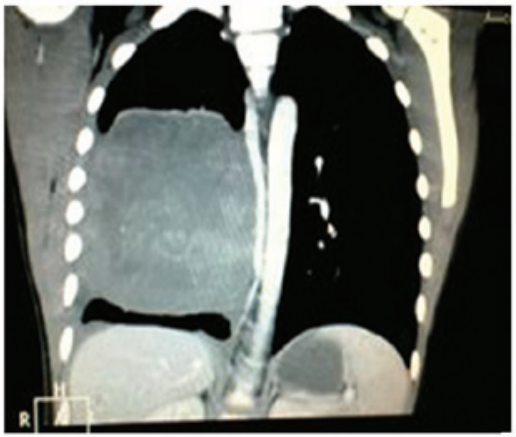

B
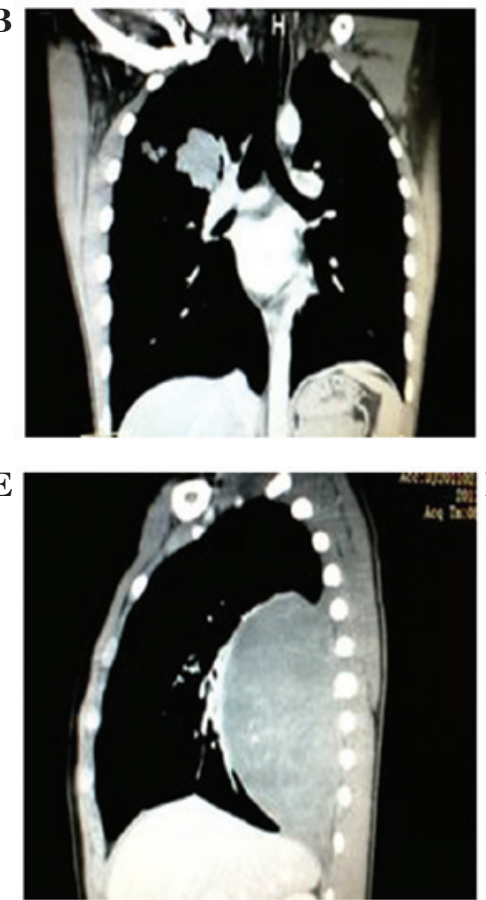
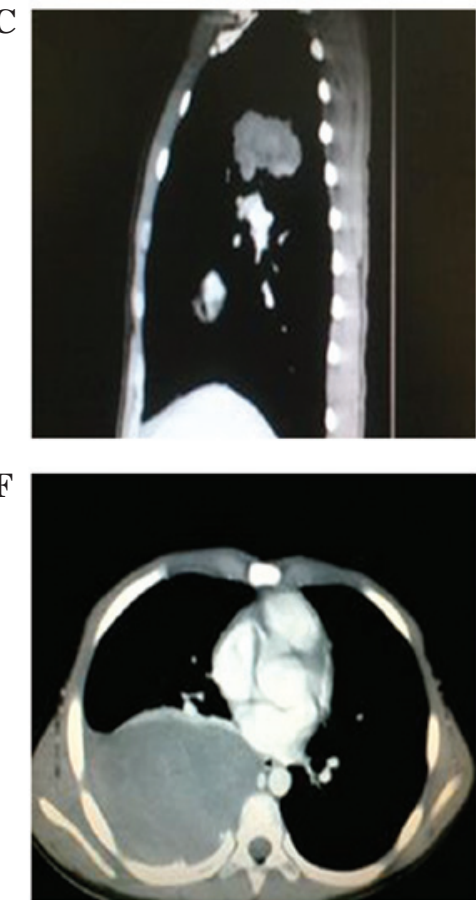

Figure 1. CT scans of two different cases. (A-C) CT scans detected a soft-tissue density mass in the upper lobe of the right lung of a patient. (D-F) CT scans detected a heterogeneous mass in the right rib cage with partial destruction of the seventh rib and pleural effusion. CT, computed tomography.

Treatment and follow-up. Eight patients underwent treatment with combined therapy, of which five underwent radical surgery with chemotherapy-radiotherapy without recurrence and three cases suffered local recurrence at 3-6 months and underwent a second surgery and post-operative adjuvant chemotherapy. These three patients underwent a second surgery and postoperative adjuvant chemotherapy. One patient only received radiotherapy, one received chemotherapy and one patient with chemotherapy-radiotherapy. Radiotherapy was performed using a $6 \mathrm{MV}$-X-ray, 3 dimensional-intensity-modulated radiation therapy dose of tumor (DT) at 40-50 Gy/20-25 fractions. Cyclophosphamide $\left(300 \mathrm{mg} / \mathrm{m}^{2}\right)$, adriamycin $\left(10 \mathrm{mg} / \mathrm{m}^{2}\right)$ and vincristine $\left(2 \mathrm{mg} / \mathrm{m}^{2}\right.$; CAV) combination chemotherapy was used for initial chemotherapy and topotecan and cisplatin (TP) combination was used for the secondary chemotherapy following recurrence. All patients were followed up for 6-24 months following treatment. The Response Evaluation Criteria in Solid Tumors (1.1) assessment criteria for physical tumor efficacy of 2009 was used to evaluate patients (5).

Statistical analysis. SPSS 13.0 software (SPSS, Inc., Chicago, IL, USA) was used for all statistical analysis. The Kaplan-Meier method was applied to calculate survival, Log-rank inspection. $\mathrm{P}<0.05$ was considered to indicate a statistically significant difference between values.

\section{Results}

Clinical features. In the present study, the common clinical symptoms of Askin's tumor include fever, cough, chest pain and suffocation, although it may present as chest lumps, swelling and pain. Certain other signs, such as short-term sickness, rapid development and plethora metastasis as well as bone and

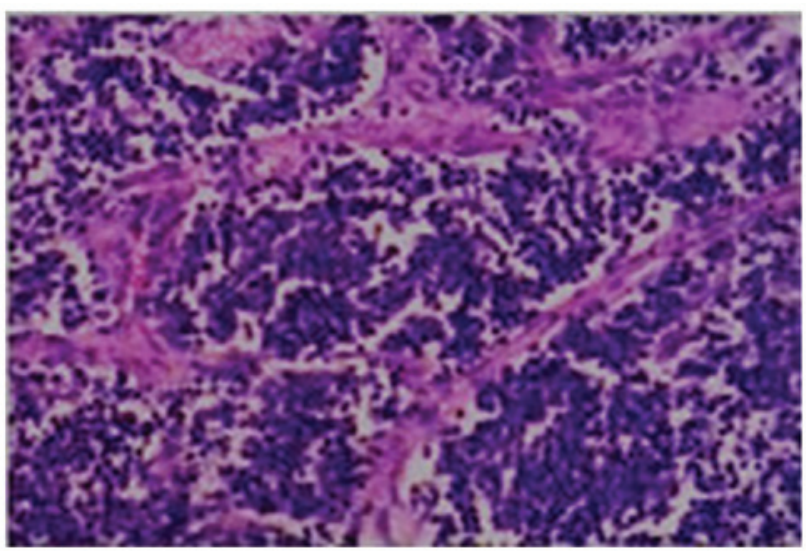

Figure 2. Representative histopathlogical examination of biopsy material demonstrating sheets of small round blue cells (magnification, x100).

lung metastasis, were also observed. Radiographic examinations revealed that the lung, pleural or chest wall do not only have larger inhomogeneous, lobulated and soft tissue masses that occasionally presented with with hemorrhage, necrosis, cystic degeneration and calcification of small needle-like tissue masses, but also have overspreading, proliferating and invasive mediastinal tissues, vertebrae or ribs.

In the present study, nine cases presented with the symptoms of fever, cough with chest pain and suffocation, while two cases presented with a mass in the chest and throat. Short-term symptoms progressed rapidly, with an average of 10-20 days. The range of Askin's tumor diameters of the seven cases was $5-12.5 \mathrm{~cm}$, as determined through CT prior to surgery. For the nine patients in which metastasis occurred, one case had pulmonary infiltrates or pulmonary metastasis and one case demonstrated clavicle and mediastinal lymph node metastasis. 
Table I. Patients with general information and analysis of prognostic factors.

\begin{tabular}{|c|c|c|c|c|}
\hline Classification & No. of cases & Proportion, $\%$ & Median survival, months & P-value \\
\hline Gender & & & & 0.306 \\
\hline Male & 3 & 27.3 & 11 & \\
\hline Female & 8 & 72.7 & 9 & \\
\hline Age, years & & & & 0.412 \\
\hline$\leq 10$ & 5 & 45.5 & 8 & \\
\hline$>10$ & 6 & 55.5 & 7 & \\
\hline Tumor size, $\mathrm{cm}$ & & & & 0.793 \\
\hline$\leq 5$ & 7 & 63.6 & 5 & \\
\hline$>5$ & 4 & 36.4 & 15 & \\
\hline Lactate dehydrogenase, U/l & & & & 0.032 \\
\hline $80-240$ & 8 & 72.7 & 12 & \\
\hline$\geq 240$ & 3 & 27.3 & 8 & \\
\hline Metastasis & & & & 0.000 \\
\hline With metastasis & 9 & 81.8 & 14 & \\
\hline Without metastasis & 2 & 18.2 & 9 & \\
\hline Therapy & & & & 0.000 \\
\hline Combined & 8 & 81.8 & 15 & \\
\hline Mono-therapy & 2 & 18.2 & 7 & \\
\hline
\end{tabular}

A further two cases were accompanied by pathological damage to the ribs and another two cases had pleural effusion. As shown in Fig. 1A-C, the patient with clavicle and mediastinal lymph node metastasis in the right upper lobe showed a lobulated mass of $5.64 \times 5.69 \mathrm{~cm}$ with no enhancement (Fig. 1A-C). In addition, surrounding the tumor were multiple heterogeneous enhancements of irregular-shaped nodular soft tissue in the right upper lung field (Fig. 1A-C). Furthermore, CT scans from another case demonstrated a soft-tissue density mass of $12.5 \times 9.5 \times 8.3 \mathrm{~cm}$ in the right rib cage with partial destruction of the seventh rib and pleural effusion (Fig. 1D-F).

Through HE staining, the morphology of biopsy specimens was observed under a microscope; the representative specimen shown in Fig. 2 was diagnosed as a single, small round-cell tumor, with neural or oval cell differentiation tendencies (Fig. 2). Of note, mitosis was common among tumor cells, fibrous stroma and blood vessels. Staining was present to a lesser degree in the cytoplasm, whereas the nucleus exhibited intense staining. The results of the immunohistochemical analysis showed that of the 11 cases, 10 (90.9\%) cases were positive for CD99 and NSE expression, while the other case was positive for NSE and S-100 expression. In addition, seven cases showed vimentin-positive expression, with negative expression for CK and LCA. These results were in accordance with the features previously reported for Askin's tumor (3).

Treatment outcomes. During the follow-up period (6-24 months), the median survival time for the combined treatment group (surgery-chemotherapy-radiotherapy) and the single treatment group (chemotherapy or radiotherapy) were 15 and 7 months, respectively. Therefore, the survival ratio was significantly improved in the combined treatment group compared with that of the single treatment group $(\mathrm{P}<0.05)$.
Univariate analysis revealed that poor prognosis was associated with a tumor diameter $>5 \mathrm{~cm}$, LDH levels $>240 \mathrm{U} / 1$ and late stage (stage III or IV) tumors. By contrast, the patient's age, gender and tumor location were not correlated with prognosis (Table I).

\section{Discussion}

Askin's tumor was firstly reported by Askin et al in 1979 (3). It was revealed that Askin's tumor shares the characteristic chromosomal translocations and has a comparable morphology, immunohistochemistry and ultrastructure with classical peripheral PNET and Ewing's tumor $(1,2)$. According to classification of tumors of the nervous system, Askin's tumor is a member of the Ewing's sarcoma family of tumors/peripheral PNETs. The clinical occurrence of Askin's tumor is rare and only a limited number of cases have been documented (6-11). The present study reported 11 cases of Askin's tumor, a relatively large sample size in terms of its incidence rate. The characteristics and treatment strategies used in the present study may therefore enhance current understanding and aid future diagnosis and treatment of Askin's tumor.

Askin's tumor may occur at any age, although it is more commonly found in children and young adults. This disease primarily occurs in the soft tissue of the chest wall, rib periosteum, chest and lung. Clinically, Askin's tumor commonly presents with the symptoms of fever, cough, chest pain, chest tightness and shortness of breath. However, symptoms may also include a chest wall mass, pleural effusion, superficial lymph nodes and rib fractures. Askin's tumor has the characteristics of a high degree of malignancy, poor efficacy and easy local recurrence. Vessel metastasis exists in the majority of cases; in addition, bone and lung metastases often occur, although 
metastases may also be transferred to brain, mediastinum, neck, mouth, liver and adrenal gland. Imaging features have been demonstrated to aid the preoperative stage diagnosis, the determination of the surgical procedure and the evaluation of treatment efficacy; however, imaging features lack specificity. Multiple magnetic resonance imaging was reported to be able to determine the source of the tumor and the scope of the chest wall invasion (12). However, CT scans appear to be more useful for the diagnosis of Askin's tumor. Typical CT scan observations for Askin's tumor include a large soft tissue mass based on the signal chest wall with or without ipsilateral pleural effusion and rib damage (9). Thus, in the present study, CT scans were performed for all patients in order to aid diagnosis.

Histopathology and immunohistochemical analysis are the basis for Askin's tumor diagnosis. Cytologic smears of the tumor revealed small round malignant cells with the typical feature of the Homer-Wright rosettes, with various layers of cells with fibrillary material $(13,14)$. For immunohistochemical analysis, the tumor was previously reported to be positive for several neural markers including NSE, CD99 and vimentin $(13,15)$. Cellular and molecular genetics studies have suggested that PNET has a characteristic chromosomal translocation t (11:22) (q24; q12), which provides an additional firm diagnostic criterion for Askin's tumor $(16,17)$. In addition, the characteristics of the disease phenotype were identified using immunohistochemical analysis, which demonstrated positive expression of CD99, NSE, S-100 and vimentin protein, while tests for CK, CgA, Syn, LCA, TTF-1 provided negative results. These results are in accordance with previous reports $(3,16,17)$.

At present, combined treatment for Askin's tumor is advised. The common procedure involves extensive resection of the tumor and surrounding tissue invading into the ribs or lungs, as well as regional lymph node dissection, followed by postoperative radiotherapy and chemotherapy. Surgical resection of the entire tumor structure and chest wall reconstruction may result in a good local control rate for thoracic lung cancer. A common regimen for radiotherapy is 40-60 Gy over 4-5 weeks and chemotherapy may include CAV, cisplatin plus etoposide or ifosfamide, etoposide plus cisplatin. However, no previous studies have reported which treatment solution is the most effective strategy for Askin's tumor. Christiansen et al (17) recommended that the most appropriate treatment plan should include preoperative and postoperative chemotherapy combined with complete surgical resection. Takanami et al (18) reported one case of a 16-year-old male patient, who suffered local recurrence and underwent six surgeries and postoperative chemotherapy; this patient was still alive at 7 years post surgery, therefore showing the importance of integrated treatment. In the present study, the combination of surgery, chemotherapy and radiotherapy resulted in optimal outcome. However, due to the number of cases and the follow-up duration, it cannot be confirmed as the most effective regimen for Askin's tumor. These results require verification from a larger number of cases in multi-center clinical trials. In conclusion, chemotherapy, treatment modalities, tumor size, tumor stage and LDH levels, among other factors may affect the survival and prognosis of patients. The development of rational and more effective strategies, such as gene therapy, based on multidisciplinary discussion may be used to improve treatment efficacy and patient prognosis.

\section{References}

1. Bunyaratavej K, Khaoroptham S, Phonprasert C, Tanboon J and Shuangshoti S: Primary intracranial peripheral primitive neuroectodermal tumor/Ewing's sarcoma presenting with acute intracerebral hemorrhage. Clin Neuropathol 24: 184-190, 2005.

2. Mobley BC, Roulston D, Shah GV,Bijwaard KE and McKeever PE: Peripheral primitive neuroectodermal tumor/Ewing's sarcoma of the craniospinal vault: Case reports and review. Hum Pathol 37: 845-853, 2006.

3. Askin FB, Rosai J, Sibley RK, Dehner LP and McAlister WH: Malignant small cell tumor of the thoracopulmonary region in childhood: A distinctive clinicopathologic entity of uncertain histogenesis. Cancer 43: 2438-2451, 1979.

4. Edge SB and Compton CC: The American Joint Committee on Cancer: The 7th edition of the AJCC cancer staging manual and the future of TNM. Ann Surg Oncol 17: 1471-1474, 2010.

5. Therasse P1, Arbuck SG, Eisenhauer EA, Wanders J, Kaplan RS, Rubinstein L, Verweij J, Van Glabbeke M, van Oosterom AT, Christian MC and Gwyther SG: New guidelines to evaluate the response to treatment in solid tumors. J Natl Cancer Inst 92: 205-216, 2000.

6. Shrestha B, Kapur BN, Karmacharya K, Kakkar S and Ghuliani R: Askin's tumor: A dual case study. Int J Pediatr 2011: 252196, 2011.

7. Sikri V and Sobti S: Askin tumour: a rare thoracopulmonary tumour in adults. Indian J Chest Dis Allied Sci 55: 233-235, 2013.

8. Benbrahim Z, Arifi S, Daoudi K, Serraj M, Amara B, Benjelloun MC, Mellas N and El Mesbahi O: Askin's tumor: a case report and literature review. World J Surg Oncol 11: $10,2013$.

9. Xu Q, Xu K, Yang C, Zhang X, Meng Y and Quan Q: Askin tumor: Four case reports and a review of the literature. Cancer Imaging 11: 184-188, 2011.

10. Gunay E, Ucar N, Aksu F, Gunay S, Orsel O, Kurt B, Taştepe I and Memiş L: A case of Askin's tumor presenting with pleural effusion and high level of adenosine deaminase. Hippokratia 15: 189-190, 2011.

11. Katsenos S, Nikopoloulou M, Kokkonouzis I and Archondakis S: Askin's tumor: A rare chest wall neoplasm. Case report and short review. Thorac Cardiovasc Surg 56: 308-310, 2008.

12. Winer-Muram HT, Kauffman WM, Gronemeyer SA and Jennings SG: Primitive neuroectodermal tumors of the chest wall (Askin tumors): CT and MR findings. AJR Am J Roentgenol 161: 265-268, 1993.

13. Kumar PV: Fine needle aspiration cytologic findings in malignant small cell tumor of the thoracopulmonary region (Askin tumor). Acta Cytol 38: 702-706, 1994.

14. Schmidt D, Herrmann C, Jurgens H and Harms D: Malignant peripheral neuroectodermal tumor and its necessary distinction from Ewing's sarcoma. A report from the Kiel Pediatric Tumor Registry. Cancer 68: 2251-2259, 1991.

15. Khoury V and Mitchell MJ: Residents' corner. Answer to case of the month \#83. Askin tumour of the chest wall. Can Assoc Radiol J 52: 269-271, 2001.

16. Downing JR, Head DR, Parham DM, Douglass EC, Hulshof MG, Link MP, Motroni TA, Grier HE, Curcio-Brint AM and Shapiro DN: Detection of the $(11 ; 22)(\mathrm{q} 24 ; \mathrm{q} 12)$ translocation of Ewing's sarcoma and peripheral neuroectodermal tumor by reverse transcription polymerase chain reaction. Am J Pathol 143: 1294-1300, 1993.

17. Christiansen S, Semik M, Dockhorn-Dworniczak B, Rötker J, Thomas M, Schmidt C, Jürgens H, Winkelmann W and Scheld HH: Diagnosis, treatment and outcome of patients with Askin-tumors. Thorac Cardiovasc Surg 48: 311-315, 2000.

18. Takanami I and Imamura T: The treatment of Askin tumor: Results of two cases. J Thorac Cardiovasc Surg 123: 391-392, 2002. 